\title{
A comprehensive gaze stabilization controller based on cerebellar internal models
}

\author{
Vannucci, Lorenzo; Falotico, Egidio; Tolu, Silvia; Cacucciolo, Vito; Dario, Paolo; Lund, Henrik Hautop ; \\ Laschi, Cecilia
}

\section{Published in:}

Bioinspiration \& Biomimetics

Link to article, DOI:

10.1088/1748-3190/aa8581

10.1088/1748-3190/aa8581

Publication date:

2017

Document Version

Peer reviewed version

Link back to DTU Orbit

Citation (APA):

Vannucci, L., Falotico, E., Tolu, S., Cacucciolo, V., Dario, P., Lund, H. H., \& Laschi, C. (2017). A comprehensive gaze stabilization controller based on cerebellar internal models. Bioinspiration \& Biomimetics, 12(6), [065001]. https://doi.org/10.1088/1748-3190/aa8581, https://doi.org/10.1088/1748-3190/aa8581

\section{General rights}

Copyright and moral rights for the publications made accessible in the public portal are retained by the authors and/or other copyright owners and it is a condition of accessing publications that users recognise and abide by the legal requirements associated with these rights.

- Users may download and print one copy of any publication from the public portal for the purpose of private study or research.

- You may not further distribute the material or use it for any profit-making activity or commercial gain

- You may freely distribute the URL identifying the publication in the public portal 
A comprehensive gaze stabilization controller based on cerebellar internal models

This content has been downloaded from IOPscience. Please scroll down to see the full text.

Download details:

IP Address: 192.38.90.17

This content was downloaded on 16/08/2017 at 09:54

Manuscript version: Accepted Manuscript

Vannucci et al

To cite this article before publication: Vannucci et al, 2017, Bioinspir. Biomim., at press:

https://doi.org/10.1088/1748-3190/aa8581

This Accepted Manuscript is: (c) 2017 IOP Publishing Ltd

During the embargo period (the 12 month period from the publication of the Version of Record of this article), the Accepted Manuscript is fully protected by copyright and cannot be reused or reposted elsewhere.

As the Version of Record of this article is going to be / has been published on a subscription basis, this Accepted Manuscript is available for reuse under a CC BY-NC-ND 3.0 licence after the 12 month embargo period.

After the embargo period, everyone is permitted to copy and redistribute this article for non-commercial purposes only, provided that they adhere to all the terms of the licence

https://creativecommons.org/licences/by-nc-nd/3.0

Although reasonable endeavours have been taken to obtain all necessary permissions from third parties to include their copyrighted content within this article, their full citation and copyright line may not be present in this Accepted Manuscript version. Before using any content from this article, please refer to the Version of Record on IOPscience once published for full citation and copyright details, as permission will likely be required. All third party content is fully copyright protected, unless specifically stated otherwise in the figure caption in the Version of Record.

When available, you can view the Version of Record for this article at:

http://iopscience.iop.org/article/10.1088/1748-3190/aa8581 


\title{
A comprehensive gaze stabilization controller based on cerebellar internal models
}

\author{
Lorenzo Vannucci ${ }^{1}$, Egidio Falotico ${ }^{1}$, Silvia Tolu ${ }^{2}$, Vito \\ Cacucciolo $^{1}$, Paolo Dario ${ }^{1}$, Henrik Hautop Lund ${ }^{2}$ and \\ Cecilia Laschi ${ }^{1}$
}

${ }^{1}$ The BioRobotics Institute, Scuola Superiore SantAnna, Viale Rinaldo Piaggio 34, 56025 Pontedera (Pisa), Italy

${ }^{2}$ Center for Playware, Department of Electrical Engineering, Technical University of Denmark, Richard Petersens Plads, Building 326, 2800 Kgs. Lyngby Copenhagen, Denmark

E-mail: lorenzo.vannucci@santannapisa.it

\begin{abstract}
Gaze stabilization is essential for clear vision; it is the combined effect of two reflexes relying on vestibular inputs: the vestibulocollic reflex (VCR), which stabilizes the head in space and the vestibulo-ocular reflex (VOR), which stabilizes the visual axis to minimize retinal image motion. The VOR works in conjunction with the opto-kinetic reflex (OKR), which is a visual feedback mechanism that allows to move the eye at the same speed as the observed scene. Together they keep the image stationary on the retina.

In this work we implement on a humanoid robot a model of gaze stabilization based on the coordination of VCR and VOR and OKR. The model, inspired by neuroscientific cerebellar theories, is provided with learning and adaptation capabilities based on internal models. We present the results for the gaze stabilization model on three sets of experiments conducted on the SABIAN robot and on the $\mathrm{iCub}$ simulator, validating the robustness of the proposed control method. The first set of experiments focused on the controller response to a set of disturbance frequencies along the vertical plane. The second shows the performances of the system under three-dimensional disturbances. The last set of experiments was carried out to test the capability of the proposed model to stabilize the gaze in locomotion tasks. The results confirm that the proposed model is beneficial in all cases reducing the retinal slip (velocity of the image on the retina) and keeping the orientation of the head stable.
\end{abstract}

Keywords: gaze stabilization, internal model, adaptive control, VOR, VCR

Submitted to: Bioinspir. Biomim. 


\section{Introduction}

Gaze stabilization is fundamental in everyday activities. The way it compensates the movements of the other part of the body as the torso, especially during basic movements like walking or running, is crucial to give a stable reference frame for the two essential perceptual systems for detection of self-motion relative to space: the visual and vestibular systems. Vision is the most useful sensor for many animals to provide information about the surrounding environment. The vestibular information operates to create an inertial guidance system determining the spatial orientation in order to coordinate movements and balance. Two kind of reflexes, which rely on the output of the inertial system and are used to keep the image stable on the retina, exist: the vestibulo-ocular reflex (VOR), which stabilizes the visual axis to minimize retinal image motion and the vestibulocollic reflex (VCR), which stabilizes the head in space through the activation of the neck musculature in response to vestibular inputs.

\subsection{VOR - From neuroscientific evidences and models to robotic controllers}

The VOR compensates for head movements that would perturb vision by turning the eye in the orbit in the opposite direction of the head movements (Barnes 1993). VOR works in conjunction with the optokinetic reflex (OKR), which is a feedback mechanism for moving the eye at the same speed as the observed scene. Together they keep the image stationary on the retina, with VOR compensating for fast movements and OKR for slower ones (Schweigart et al. 1997). The VOR involves six extraocular muscles, each pair acts around a single rotation axis. When a rotation of the head is detected, the semicircular canals get activated by head rotation and send their impulses via the vestibular nerve, through the vestibular nuclei, to the extraocular muscles (an inhibitory signal to the muscles on one side and an excitatory signal to the muscles on the other side). The result is a compensatory movement of the eyes. The neural control circuitry of the horizontal VOR has been studied intensively since it involves only three neuron reflex arc and the cerebellar flocculus (Itō 1984) which is also known to be involved in the OKR. The VOR is an adaptive mechanism which changes with experience. The vestibulocerebellum is considered to be responsible for this learning. Several studies confirm that the cerebellum is essential for motor learning to correct the VOR in order to ensure accurate eye movement. To test this hypothesis, Ito and colleagues (Ito et al. 1977) studied the influence of a stimulation applied to the inferior olivary or to the optic tract to the vestibulo-ocular reflex. In healthy rabbit, an olivary stimulation induced a depression of the discharge sent by the semicircular canals. In parallel with the electrophysiological studies, authors tested the effect of a lesion on the VOR. Using spectacles that reversed left and right direction on cats, Robinson (Robinson 1976) showed that cats are able to adapt their VOR gain to achieve a stable vision. When the flocculus, paraflocculus and some parts of the vermis were removed, Robinson (Robinson 1976) reported no more long- or short-term adaptation. An interesting hypothesis on cerebellar motor learning, based on an experimental study of the rabbit cerebellum, was proposed by Ito (Ito 2000) who followed the Marr-Albus model (Marr \& Thach 1991, Albus 1971). According to Ito, the adjustment of the relative strengths of the direct excitatory and indirect inhibitory pathways adaptively modulates the gain of the VOR. Ito asserted that the modulation could be influenced by messages of retinal image slip and the role of the cerebellum is to store the motor memory for the changes in VOR gains. Miles and Lisberger (Miles \& Lisberger 1981), proposed an alternative model. They stated that the role of the cerebellum was not to store memories but rather to compute an instructive signal to guide the plasticity process.

One of the first neuroscientific models of VOR has been proposed by Schmid et al. (Schmid et al. 1971) based on human recordings with the aim to compare the frequency response of their model to the human behavior. Galiana and Outerbridge (Galiana \& Outerbridge 1984) published a bilateral model of the VOR based on the anatomy of the main neuronal connections present in the central VOR pathway. The model can reproduce the activity of the different populations of neurons when a subject is rotating in the dark. Gomi and Kawato (Gomi \& Kawato 1992) proposed a model based on the simultaneous adaptation of VOR and OKR, and compared it with the biological data. Finally, Green and Angelaki (Green \& Angelaki 2004) proposed model based on a neural network that combines the sensory information from otoliths and semicircular canals simulating the response along the three rotational axes (yaw, pitch and roll). Other models focus on the visual-vestibular interaction ((Lau et al. 1978) (Schmid et al. 1980) (Barnes 1993). Lau and colleagues (Lau et al. 1978) published a VOR model with a linear interaction between the vestibular and the visual component. With this simple model, they simulated the behaviour of the VOR in the dark, in the light, with a headfixed target and with a target oscillation added to the scillating chair. The model of Lau et al. (Lau et al. 1978) was built to reproduce all the experimental conditions for a single oscillation frequency of $0.05 \mathrm{~Hz}$. After that, Schmid et al. (Schmid et al. 1980) updated 
the model of Lau et al. (Lau et al. 1978) and integrated non-linear elements to better represent the behaviour of the visual-vestibular interaction on a larger range of target velocities. Barnes (Barnes 1993) proposed a model that includes three loops to reproduce the visual modulation during pursuit and the non-visual modulation of the VOR. In this model, a central control triggers either an enhancement or a decrease of the VOR response to model a volitional control of the VOR. All these models aim to validate human experiments or try to replicate the neural circuits involved in this tasks, but are not suitable for the implementation on robotic platforms.

Two biological models of the VOR have been proposed and tested on robotic platforms. Shibata and Shaal (Shibata \& Schaal 2001) propose to use a feedback error learning strategy in conjunction with a receptive field weighted regression algorithm to implement the VOR and its internal model on a humanoid robot. Porrill and colleagues (Porrill et al. 2004) presented a model for the VOR that is capable of working on three rotational axes and that includes an internal model and provide an implementation on Matlab. This model has also been implemented on a robotic platform simulating the eye movements (Lenz et al. 2008) In robotics literature we found some other controllers inspired by the VOR (Viollet \& Franceschini 2005, Franchi et al. 2010). Viollet and Franceschini developed a gaze controller that includes a feedforward component inspired by the VOR, but lacking any sensory-motor corrective module. Finally, in (Franchi et al. 2010), a decorrelation model replicating the VOR, that is implemented with a Recursive Least Square algorithm, is shown to be working on a robotic simulator. Panerai and colleagues presented a bio-inpired controller based on an inertial sensory apparatus and images of space-variant resolution (Panerai \& Sandini 1998, Panerai et al. 2000). Among all these controllers, only Shibata and Schaal (Shibata \& Schaal 2001) replicated the OKR mechanism and showed the cooperation between ocular movements.

\subsection{VCR - From neuroscientific evidences and models to robotic controllers}

The VCR stabilizes the head based on the inertial input space by generating a command that moves the head in the opposite direction to that of the current head-in-space displacement. When the head is rotated in the plane of a semicircular canal, the canal is stimulated and the muscles are activated. Thanks to this stimulation, a compensatory rotation of the head along the same axis is produced. Each canal activation produces an appropriate reflex response. The VCR controls a complex musculature that includes more than 30 muscles controlling pitch, roll and yaw rotations (Peterson et al. 2001). The system could be considered underdetermined because there are more muscles than rotation axes. One consequence of this property is that the same head movement can be produced by the activation of different muscle patterns: this is the case for voluntary head movements, but not for the VCR where a particular head motion is related to a stereotyped muscle activation pattern (Peterson et al. 2001).

The neuroscientific studies conducted on human patients during locomotion evidence the contribution of the VCR for the head stabilization. During a normal straight walking the head moves mainly in the sagittal plane, rotating around the pitch axes in a range of $0.3-8^{\circ}$ and translating between $0.8-$ $9 \mathrm{~cm}$ (Pozzo et al. 1990, Bloomberg et al. 1992). During fixed-gaze treadmill walking, that is at fixed velocity, the coordination between the head and the trunk is dependent on the events in a gait cycle (Mulavara et al. 2002), although the trunk already damps many oscillation acting as a low pass filter (Kavanagh et al. 2006), the role of the reflexes is still essential. The neural pathways mediating this reflex are as yet uncertain. Despite this, the involvement of the cerebellum in the vestibulo spinal reflexes, including the VCR, is well known (Cullen \& Roy 2004, Manzoni et al. 1994). For a complete review about the role of vestibular system in posture control and involvement of the cerebellum in stabilization tasks refer to (Cullen 2012). Task involving visual fixation lead to an improvement of head-in-space stabilization in humans (Goldberg \& Cullen 2011) due to increased VCR contributions (Forbes et al. 2013). Rather, it is still unclear whether a suppression of the VCR occurs in voluntary head movements (Forbes et al. 2014). Although there are many physiological models of the VOR and many robotic implementations, it is not really the same for the VCR. The existent models have incomplete characteristics or are not suitable for a robotic implementation. The current state of art provides two main important models for the aforementioned reflex. Both consider the VCR as a simple negative feedback, but recent studies (Forbes et al. 2013) demonstrates the engagement of the central nervous system in the modulations of feedback gains. This means that the simple feedback is not enough to explain the mechanisms involved in head stabilization and that some feedforward contribution is present. A model of VCR has been proposed by Peng (Peng et al. 1996) in 1996. They presented a control model of yaw head rotations during perturbations of the torso, which for the first time combine a model of the human head with neural feedback controllers representing the vestibulocollic and the cervicocollic reflexes. The parameters of the model are extracted directly from 
anthropomorphic, biomechanical and physiological studies. The model proposed by Goldberg and colleagues (Goldberg \& Cullen 2011) is nearly the same as the previous one but in addition there is the contribution of the voluntary control.

In robotics, some head stabilization models already exist. Yamada and colleagues (Yamada et al. 2007) proposed a method for controlling the neck of a snake-like robot in order to stabilize the head against undulations. The controller is based on the rejection of the disturbance of the body on the head using a continuous model. The model proposed by Marcinkiewicz (Marcinkiewicz et al. 2009), implemented on the AIBO robot, uses a machine learning algorithm able to learn how to compensate for head rotations in the absence of stabilization mechanisms. Gay and colleagues (Gay et al. 2013) proposed a head stabilization system for a bipedal robot during locomotion controlled by the optical flow information. It is based on Adaptive Frequency Oscillators to learn the frequency and phase shift of the optical flow. Although the system can successfully stabilize the head of the robot during its locomotion, it does not take in consideration the vestibular inputs.

The closest to the neuroscientific findings of the VCR are the works proposed by Kryczka and colleagues (Kryczka et al. 2012b, Kryczka et al. 2012a, Falotico et al. 2012). They proposed an inverse jacobian controller (Kryczka et al. 2012b, Kryczka et al. 2012a) based on neuroscientific results (Falotico et al. 2011) and an adaptive model based on a feedback error learning (FEL) (Falotico et al. 2012) able to compensate the disturbance represented by the trunk rotations. A comparison between these models of head stabilization is provided in (Falotico et al. 2017).

\subsection{Objectives and rationale}

All the presented models try to reproduce specific aspects of the gaze stabilization behaviour, but none of them can provide a comprehensive model of gaze stabilization, integrating eye stabilization (OKR and VOR) together with head stabilization (VCR). Some robotic controllers provide mechanisms involving eyes and head, but they usually consider a unique kinematic chain (neck-eyes) with the aim of stabilize the image on the retina (Roncone et al. 2014, Habra \& Ronsse 2016) without any learning mechanism involved. With the aim of providing an efficient regulation of the gaze system, in this work we present a complete model of gaze stabilization based on the coordination of VCR and OKR/VOR (Vannucci, Tolu, Falotico, Dario, Lund \& Laschi 2016, Vannucci, Falotico, Tolu, Dario, Lund \& Laschi 2016). By inspiration on the aforementioned cerebellar theories, an adaptive learning mechanism is integrated in the model. This model is tested on a humanoid robot on three sets of experiments. The first set of experiments focused on the controller response to a set of disturbance frequencies along the vertical plane. The second shows the performances of the system under threedimensional disturbances. The last set of experiments was carried out to test the capability of the proposed model to stabilize the gaze in locomotion tasks.

Moreover, in addition to showing how the adaptive learning mechanism can be used to improve the performances of gaze stabilization while executing a specific task that is long enough to allow for a proper learning phase, it is shown how such mechanism can be employed to perform learning even when the trials are short or to create a more general controller capable of adapting to various execution tasks. This is implemented through an offline learning technique that allows to create training sets, upon which learning is performed, and then to store the internal model parameters after learning for later use in a modified version of the stabilizing controller. This also allows the proposed method to be used in short, but repeatable (up to a certain degree of similarity) task, such as biped locomotion.

This is the first time that such a comprehensive control model, with its offline learning capability, is employed for the gaze stabilization on a physical humanoid robot.

\section{Gaze Stabilization controller}

The VOR-OKR-VCR stabilization system is based on the model proposed in (Vannucci, Tolu, Falotico, Dario, Lund \& Laschi 2016, Vannucci, Falotico, Tolu, Dario, Lund \& Laschi 2016). Such model comprises feedforward and feedback controllers and learning networks that provide corrective control signals through internal models. The overall architecture of the controller is shown in Figure 1.

\subsection{Internal model}

The implementation of the internal model aims at replicating the functionalities of the cerebellum and was proposed in (Tolu et al. 2012, Tolu et al. 2013). It comprises a machine learning algorithm, Locally Weighted Projection Regression (LWPR) (Vijayakumar \& Schaal 2000), that models the cerebellar granular layer (mossy fibers and granule cells), and a linear readout modelling the integration of information performed by the Purkinje cells, denoted as Purkinje Layer (PL). Both the LWPR algorithm and the linear readout can be trained by feedback signals (climbing fibers). Given the control architecture of the subsystems, the internal models act as inverse dynamic models that take as an input the reference and the 


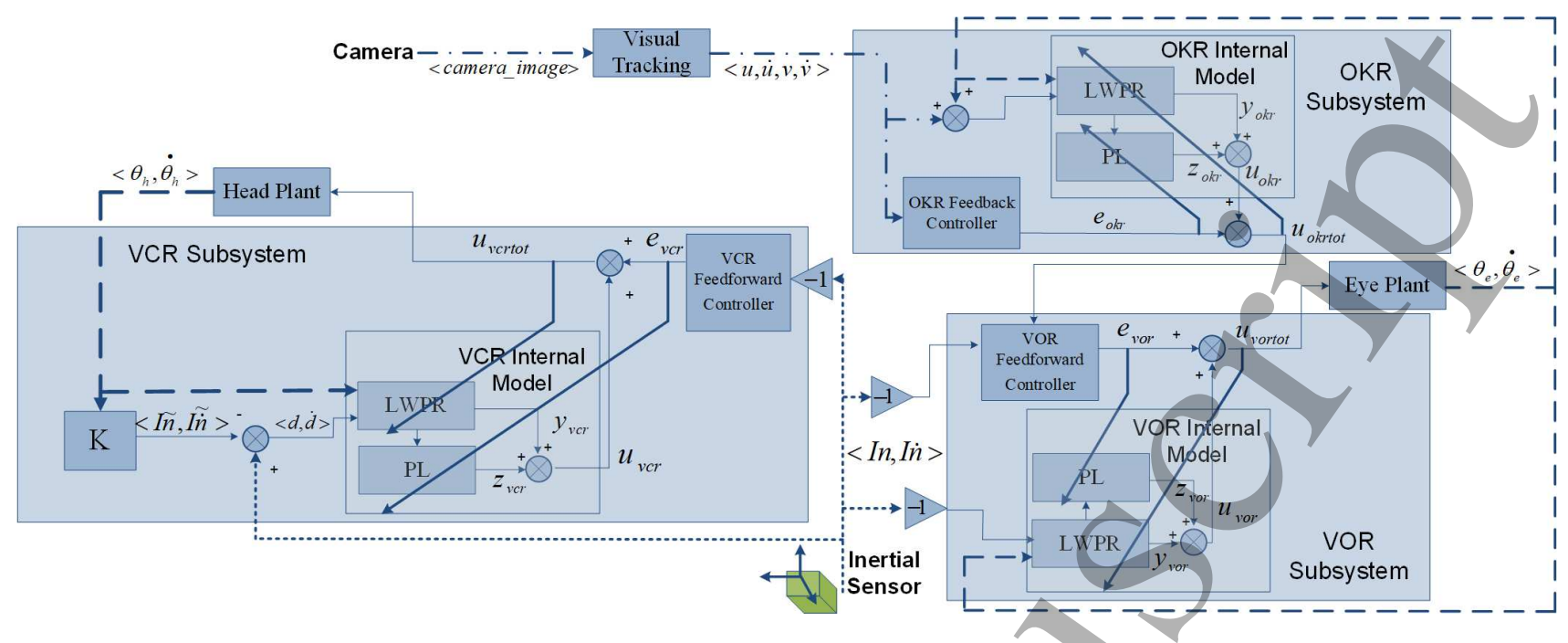

Figure 1: The proposed Gaze Stabilization controller. All three subsystems consist of a feedforward or feedback controller and the relative internal model, whose components, LWPR and PL, contribute in the generation of the motor commands, by providing appropriate corrections. The training signals for the PL and LWPR are the output of the feedforward or feedback controller and the total motor command, respectively.

current state of the robot, and produce the desired motor command that minimize the output of the feedforward or feedback component (Tolu et al. 2012). This, in turn, is dependent on both the sensory delays and the robot dynamics itself, so the internal model is able to provide motor commands that compensate for these delays, therefore improving the performances of stabilization system.

The LWPR is a non-linear regression model that divides the input space into a set of receptive fields. Each one has a centre $c_{k}$ and an extension defined by a positive definite distance matrix $D_{k}$. The activation of each receptive field $k$ in response to an input $x$ and its output are expressed by

$p_{k}(x)=\exp \left(-\frac{1}{2}\left(x-c_{k}\right)^{T} D_{k}\left(x-c_{k}\right)\right)$,

$y_{k}(x)=w_{k} \cdot x+\epsilon_{k}$,

where $w_{k}$ and $\epsilon_{k}$ are the weight vector and bias associated with the $k$-th linear model. With every new input, the centre and the distance matrix associated with the assigned receptive field are updated.

The global output of the LWPR is given by the weighted mean of all the outputs $y_{k}$ of the linear local models created:

$$
y(x)=\frac{\sum_{k=1}^{N} p_{k}(x) y_{k}(x)}{\sum_{k=1}^{N} p_{k}(x)} .
$$

By employing localized linear models, the LWPR algorithm lowers the computational cost and enable the possibility for online, real-time learning.

The activation response of the LWPR, $p_{k}$, is used to compute the output of the Purkinje Layer:

$$
z(x)=\sum_{k} r_{k} p_{k}(x)
$$

where $r$ is a set of weights that can be trained with an update rule derived from (Porrill \& Dean 2007):

$\delta r_{k}=-\beta e(x) p_{k}(x)$,

where $\beta$ is a positive learning rate and $e(x)$ is the error signal for the current input.

The output of the internal model is the sum between the output of the LWPR and of the PL:

$u(x)=y(x)+z(x)$.

\subsection{Vestibulocollic reflex subsystem}

In this subsystem, the feedforward controller $\left(e_{v c r}\right)$ provides velocity motor commands that stabilize the head against disturbances originated by torso movements. The output of the internal model $\left(u_{v c r}\right)$ is added to the motor command in order to provide corrective sensory-motor signals, thus increasing the performances of the system. The VCR Feedforward Controller is implemented as a PD controller, and its output is computed as a function of the inertial readings (orientation as Euler angles, In, and rotational velocities, $\dot{I n})$ :

$e_{v c r}=k_{p} \cdot(-I n)-k_{d} \cdot(-\dot{I n})$.

Albeit the choice of using a PD controller to model the Feedforward component of the reflexes is not biologically inspired, in this work we aim at a robotic application, therefore such simplification can still be considered appropriate in this context. The internal 
model receives as input the current and the desired angular position and velocity of the robot head, and it is trained with the output of the feedforward controller and the resulting motor command. The desired angular position and speed can be computed as the current intensity of the external disturbance. This can be estimated using readings coming from the inertial measurement unit and the encoder values, using only direct kinematics functions. The disturbance vector can be computed as $d=I n-\tilde{I n}$, i.e. by subtracting the expected angular rotations given by the encoder values $(\tilde{I n})$ from the inertial readings $($ In $) . \tilde{I n}=[\varphi, \vartheta, \psi]$ are the Euler angles for the rigid roto-translation matrix $K\left(\theta_{h}\right)$ from the root reference frame to the inertial frame, computed as:

$\varphi=\operatorname{atan} 2\left(-K\left(\theta_{h}\right)_{2,1}, K\left(\theta_{h}\right)_{2,2}\right)$,

$\vartheta=\operatorname{asin}\left(K\left(\theta_{h}\right)_{2,0}\right)$,

$\psi=\operatorname{atan} 2\left(-K\left(\theta_{h}\right)_{1,0}, K\left(\theta_{h}\right)_{0,0}\right)$.

The angular velocity of the disturbance can be estimated as follows:

$\dot{d}=\dot{I n}-\dot{\tilde{I n}}=\dot{I n}-J\left(\theta_{h}\right) \cdot \dot{\theta_{h}}$,

where $J$ is the geometric Jacobian from the root reference frame to the inertial frame.

\subsection{Vestibulo-ocular reflex subsystem}

The VOR subsystem receives in input the head angular position and velocity signals, acquired through the inertial measurement unit $(I n, \dot{I n})$, and use them to generate appropriate velocity motor commands that compensate for head motion. As for the VCR subsystem, it comprises of a feedforward controller, implemented as a PD, and an internal model. The output of the PD controller can be computed as

$e_{v o r}=k_{p} \cdot(-I n)+k_{d} \cdot(-\dot{I n})+u_{\text {okrtot }}$,

where $u_{\text {okrtot }}$ is the output of the OKR subsystem, employed as compensatory signal (Shibata \& Schaal 2001). The VOR internal model receives as inputs the desired eye rotations (i.e. inertial angular positions and velocities), along with the current eye encoder values $\left(\theta_{e}\right.$ and $\left.\dot{\theta_{e}}\right)$. As in the VCR subsystem, the learning signals are the output of the feedforward controller and the generated motor command.

\subsection{Opto-kinetic reflex subsystem}

The position error of the visual target, computed as its angular position on the horizontal and vertical axes in the camera reference frame $(u, v)$, and the retinal slip, its derivative $(\dot{u}, \dot{v})$ are used by the OKR subsystem to keep the camera image stable. The angular position is extracted from the camera image using colour filtering, centroid computation and a conversion from pixels to angles. This information is used to compute the output of the feedback controller:

$e_{o k r}=k_{p} \cdot(u, v)+k_{d} \cdot(\dot{u}, \dot{v})$.

As in the previous cases, the learning signals for the OKR internal model are the output of the feedback controller and the generated motor command. The inputs to the model are the current eye state and the sum of the eye position and velocity and the tracked target position and velocity, that are the absolute value of the camera image motion in the eye reference frame.

\subsection{Offline learning}

While the proposed model is capable of online, realtime learning, this is not suitable for every situation. In fact, once the internal model has been learnt, it could be used on subsequent trials in order to avoid the training phase. Moreover, the training could be performed offline, either by collecting a set of trials that can be used as a training set or by generating a random disturbance. The first approach can be useful to train the controller for a specific task which is too short to allow online learning, while the second one can be employed to expose the controller to a wider range of disturbances, creating a more generic, adaptive controller which can be later employed under different disturbances. Once the learning phase is over, the LWPR and PL parameters can be stored and used in a new trial in order to avoid having to perform the learning again. In particular, the parameters that have to be saved for the LWPR are the number of receptive fields, the distance matrices $D_{k}$, the weight vectors $w_{k}$ and the biases $\epsilon_{k}$, while for the PL the vector of weights $r$ must be saved.

After the learning phase, the contribution of the non internal model term (feedforward or feedback controller) to the resulting motor command tends to be null (Tolu et al. 2012). Thus, in the offline learning case, we did not use the feedback controllers for the subsystems during the testing trials, simplifying the control scheme as shown in Figure 2.

\section{Experimental setup}

In order to assess the performances of the proposed model, three different kinds of experiments have been performed:

- stabilization of artificially generated disturbances;

- stabilization of 3D disturbances;

- stabilization of locomotion disturbance.

In the first case, the iCub robotic head was mounted on a oscillating platform capable of generating disturbances through periodic motion (see Figure 


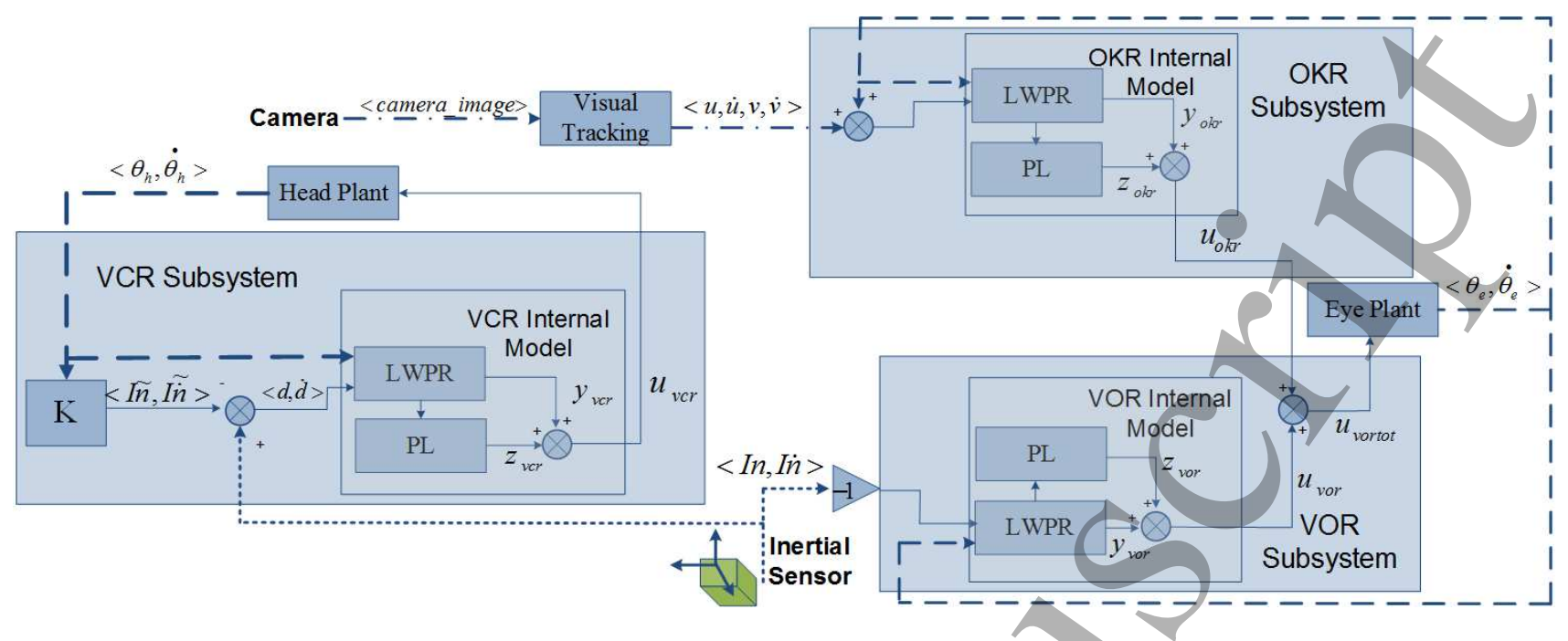

Figure 2: The modified Gaze Stabilization for the execution after alearning phase.

3a). The iCub head (Beira et al. 2006) has 6 degrees of freedom: 3 for the neck (pan, tilt and swing) and 3 for the eyes (an independent pan for each eye and a common tilt). All the joints are actuated by DC servo motors with relative encoders. The visual stereo system consists of 2 dragonfly2 cameras with a maximal resolution of 320x240 pixels and a frame rate of $60 \mathrm{~Hz}$. The Inertial Measurement Unit (IMU), mounted in the centre of the head, is an XSens MTx unit. Inertial measurements, with an angular accuracy on the roll and pitch axis of 2 degrees can be sampled with a $100 \mathrm{~Hz}$ frequency.

The oscillating platform is a custom built device that generates periodic disturbances and it consists in an aluminium plate connected to a rigid external frame by two coaxial bearings. The device to be tested is placed on the plate and anchored mechanically. The plate is then connected to a rigid mechanism that transforms the continuous rotation of a crank, actuated by a DC motor, through a slide mechanism, to the periodical oscillation of the plate (Figure 3b). The platform can only provide disturbances on a single rotational axis, and the rotation of the plate $(\gamma)$ can be expressed as a function of the crank angle $(\delta)$ :

$\sin (\gamma)=\frac{R \cdot \sin (\delta)}{\sqrt{(R \cdot \sin (\delta))^{2}+(L-R \cdot \cos (\delta))^{2}}}$,

where $R$ is the distance from the motor axis and the pin joint linking the disk and the slider and $L$ is the distance between the axis of the motor and the axis of rotation of the platform. By construction these parameters are $/$ set to $R=2 \mathrm{~cm}$ and $L=20 \mathrm{~cm}$.

Due to the limitations of the platform, that provides disturbances on a single rotational axis, only the pitch axis could be considered for the experiments, thus only rotations around the y axis of the inertial reference frame.
In order to verify that the controller is capable of working also for disturbances on all rotational axes, experiments were also performed on the iCub robotic simulator (Tikhanoff et al. 2008). In this case, the disturbance is provided by moving the robot torso.

For the stabilization of locomotion disturbance, we employed the SABIAN humanoid robot in a straight walking task. This robot has an iCub robotic head mounted on the body of the WABIAN-2 humanoid robot (Ogura et al. 2006), that has 7 DOF in each leg and $2 \mathrm{DOF}$ in the waist, with a bio-inspired range of motion. These make the robot able to perform humanlike walking, with stretched knees and raising the hip, in contrast with most humanoid robots that walk with the knees bent.

As the iCub head encoders do not provide velocity information, a smooth differentiation technique was employed to compute the velocity of joints. This introduced a sensory delay of around $100 \mathrm{~ms}$. To align all sensory feedback, we also delayed position, inertial and visual information accordingly.

\section{Results}

During all the experiment the main measure of error considered is the movement of the camera image (retinal slip). Human vision is considered stable if such error is below $4 \mathrm{deg} / \mathrm{s}$ (Collewijn et al. 1981). In order to compute the retinal slip, and to generate the input for the OKR subsystem, a target was placed in the field of view of the robot camera. The other measure of performance considered is the inertial orientation of the head, read through the IMU. 

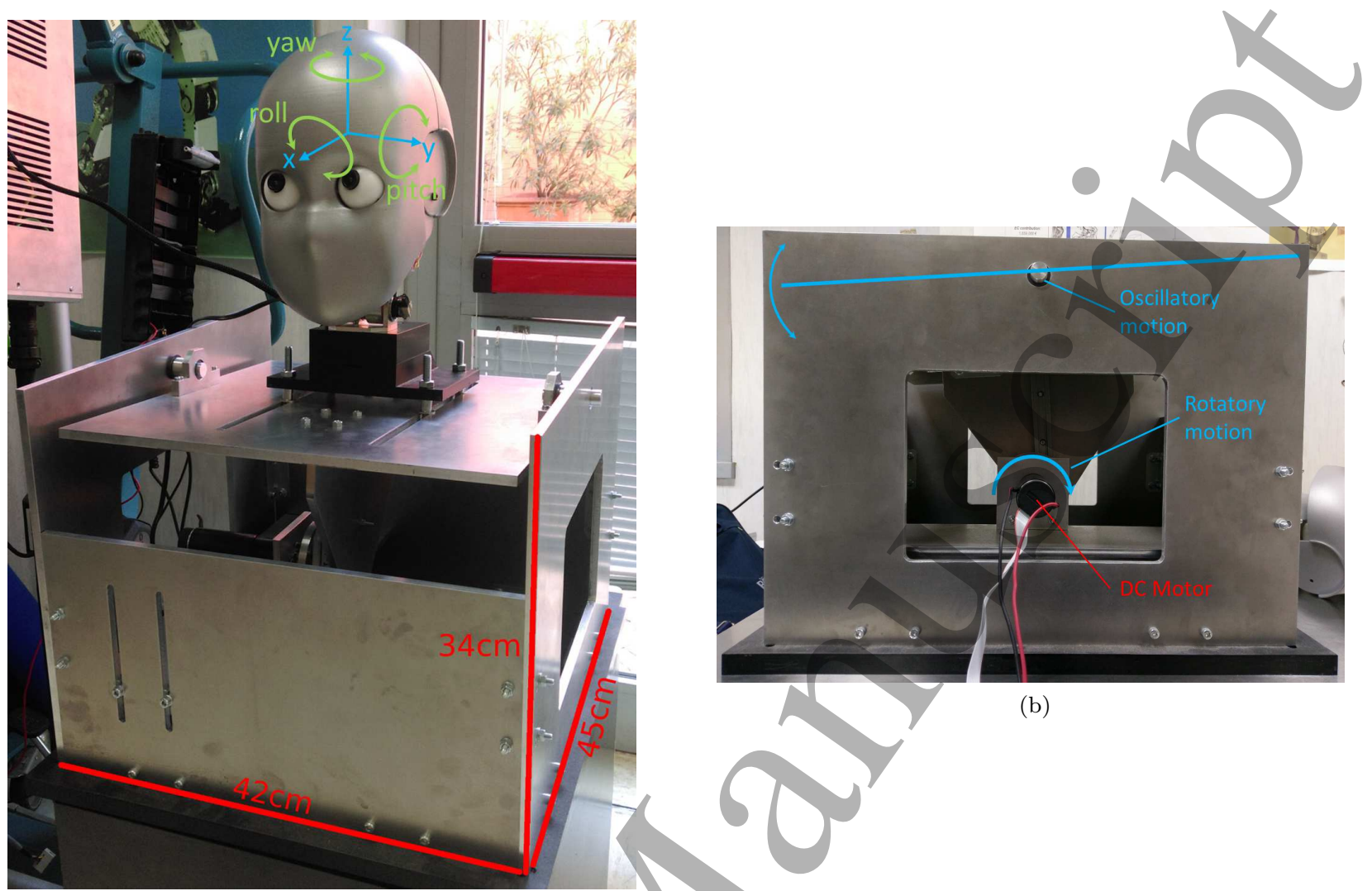

(a)

Figure 3: The oscillating platform. In (a) the iCub head mounted on the platform, with its inertial reference frame is shown. The transmission of motion from the DC motor to the oscillating platform is depicted in (b).

\subsection{Stabilization on the oscillating platform}

During these trials, the robotic head was mounted on the oscillating platform that was controlled in order to produce different disturbance motions. In particular the frequency of the periodic disturbance $(f)$ was varied during the tests.

In a first set of trials, due to their length and repeatability, no information about the learnt internal models was retained, thus the online learning phase of the LWPR and the linear readout always started from scratch at the beginning of the trial. During these tests, the frequency of motion was changed in an increasing fashion, thus providing trials of increasingly disturbance speed. During these trials, the target was kept static in front of the platform. Due to the target being static, the OKR subsystem does not provide substantial contributions during these trials, thus it was disabled. This also improves learning times, as the OKR output can slow down the learning, especially at high frequencies. Results for $f$ ranging from $0.3 \mathrm{~Hz}$ up to $1.5 \mathrm{~Hz}$ can be found in Table 1 where, for each frequency, the Root Mean Square (RMS) value of the disturbance speed $\dot{d}_{p}(\mathrm{deg} / \mathrm{s})$, inertial sensor readings,
$I n_{p}(\mathrm{deg})$ and $\dot{I n}_{p}(\mathrm{deg} / \mathrm{s})$, and the position and velocity of the target on the camera image on the vertical axis, $v(\mathrm{deg})$ and $\dot{v}(\mathrm{deg} / \mathrm{s})$, are presented. In order to avoid having the results influenced by the instability of the initial learning phase, the RMSs were computed only on the last 5 of the 14 total seconds of the trials. It can be observed that the controller is able to stabilize the image on the camera against disturbances generated by motion frequencies up to 1Hz. After that, the retinal slip $(\dot{v})$ increases over $4 \mathrm{deg} / \mathrm{s}$. However, by comparing the magnitude of the disturbance and the retinal slip, it emerges that the controller is able to reduce it to one third even for frequencies up to $1.4 \mathrm{~Hz}$, thus providing a substantial contribution towards a stable camera image. An example of such trials (for $f=1 \mathrm{~Hz}$ ) can be seen in Figure 4. It can be observed that, after an unstable learning phase at the beginning, the magnitude of the error is reduced dramatically over time, in both the velocity and position spaces. At the same time, the control signal coming from the internal model, especially from the LWPR component, increases, while the output of the feedforward controller (FFC) is reduced towards zero. This implies a switch to a 
sensory-motor compensatory motion that is able to almost nullify the disturbance.

Table 1: Results for disturbances provided by the oscillating platform at various frequencies.

\begin{tabular}{|c||c|c|c|c|c|}
\hline$f$ & $\dot{d}_{p}$ & In $_{p}$ & In $_{p}$ & $v$ & $\dot{v}$ \\
\hline 0.30 & 9.46 & 0.32 & 1.16 & 0.29 & 0.94 \\
0.50 & 15.79 & 0.17 & 1.40 & 0.30 & 1.06 \\
0.70 & 18.58 & 0.18 & 1.78 & 0.34 & 1.01 \\
0.85 & 22.54 & 0.32 & 3.33 & 0.48 & 0.99 \\
1.00 & 25.66 & 0.78 & 7.31 & 1.15 & 2.20 \\
1.20 & 29.62 & 0.78 & 11.17 & 2.61 & 7.94 \\
1.40 & 34.98 & 1.21 & 15.74 & 2.06 & 9.44 \\
1.50 & 39.70 & 2.59 & 28.68 & 3.55 & 27.16 \\
\hline
\end{tabular}

It is also relevant to stress the importance of the combination of VCR and VOR subsystems during these tasks: in fact, as shown in Table 1, while the VCR subsystem is able to provide a substantial contribution towards stabilization, reflected in the low inertial readings RMS values, it is only thanks to the added contribution of the VOR subsystem that full stabilization is achieved in some cases. Moreover, if employed separately, the VCR and VOR subsystems cannot achieve the same performances, as depicted in Figure 5, where the results for trials where only one of the two subsystems was active are shown. It can be observed that each of the two subsystems work and is able to reduce the error generated by the disturbance. However, the RMS value for the retinal slip is respectively $7.86 \mathrm{deg} / \mathrm{s}$ and $4.01 \mathrm{deg} / \mathrm{s}$ for the VOR and VCR subsystems. Thus, only with the combination of the two, a stable camera image is ensured.

In order to simulate the drifting motion that elicits the OKR response, the target, placed in front of the platform, was moved with a sinusoidal motion on the vertical axis with a frequency of $0.15 \mathrm{~Hz}$ and a peakto-peak amplitude of $15 \mathrm{deg}$ in the camera reference frame, while the frequency of the disturbance was kept at $1.0 \mathrm{~Hz}$. The behaviour of the system during this trial is shown in Figure 6. It can be noticed that the controller is able to stabilize the image on the camera and almost no trace of the target movement can be observed in the position or velocity space plots. The RMS value of the retinal slip in this trial is $3.69 \mathrm{deg} / \mathrm{s}$, thus the camera image is kept stable, even if the error is increased, compared to the trial with a static target.

In a second set of tests, we employed the offline learning strategy to create a more general controller that can work on different disturbances. To achieve this, we performed a training phase of 150 s during which the platform was moved with a sinusoidal signal whose frequency randomly varied over time. The motion was generated by varying the voltage applied to the motor using a colored noise signal (low-pass filtered white noise) with a maximum voltage of $3 \mathrm{~V}$. This led to frequencies of motion up to 1.2Hz. Figure 7 shows the behaviour of this learning phase. It can be observed that, after an initial phase of circa 50 seconds where the learning occurs, the RMS of the inertial and camera measures, averaged over time bins of $3 \mathrm{~s}$, remain stable and no further improvements are noticeable. By performing a similar trial with the, learnt internal models (Figure 7, bottom row), we can observe that initial unstable phase has disappeared and that the disturbance is immediately compensated. After learning, we employed the controller with the learnt internal models on a test trial in which a sinusoidal signal whose frequency gradually increased every 5 seconds was applied to the platform. Figure 8 shows the behaviour of the RMS during such trial, averaged over time bins of $1 \mathrm{~s}$. Being already trained, the system is able to immediately stabilize the disturbance and the performances do not significantly decrease when the signal changes frequency. By comparing the behaviour of the same task in the online learning scenario, it can be noticed that in the latter case the performances are inferior as the system needs to learn after each frequency change and it has not enough time to properly do so. Therefore, a long offline training phase provides a viable solution to create more general controllers capable of adaptation to different tasks.

\subsection{Stabilization of $3 D$ disturbances}

Due to the movements of the oscillating platform being limited to only one rotational axis, stabilization of disturbances applied on all three axes could only be done in simulation.

In these tests, sinusoidal disturbances were applied to the three joints of the torso to produce a threedimensional disturbance. In this case, the VCR is able to provide stabilizing motor commands on all three rotational axes, while VOR and OKR can only stabilize on the pitch and yaw rotational axes, as the robot eye joints do not rotate around the roll axis. Results for $f$ ranging from $0.3 \mathrm{~Hz}$ up to $1.5 \mathrm{~Hz}$ can be found in Table 2, where the same terminology of Table 1 is used and $r, y, u$ are the roll axis, yaw axis and horizontal axis of the camera image, respectively. It can observed that, while the inertial reference frame is stabilized by the VCR, the image on the camera appear to be not stable. This is because the combination of all the three rotational movements produces a linear motion of the head in space, whose effects cannot be canceled by the controller, as linear measurements are not taken into account as inputs. This motion is particularly evident on the horizontal axis. This disturbance can only be partially canceled by the OKR, especially at 

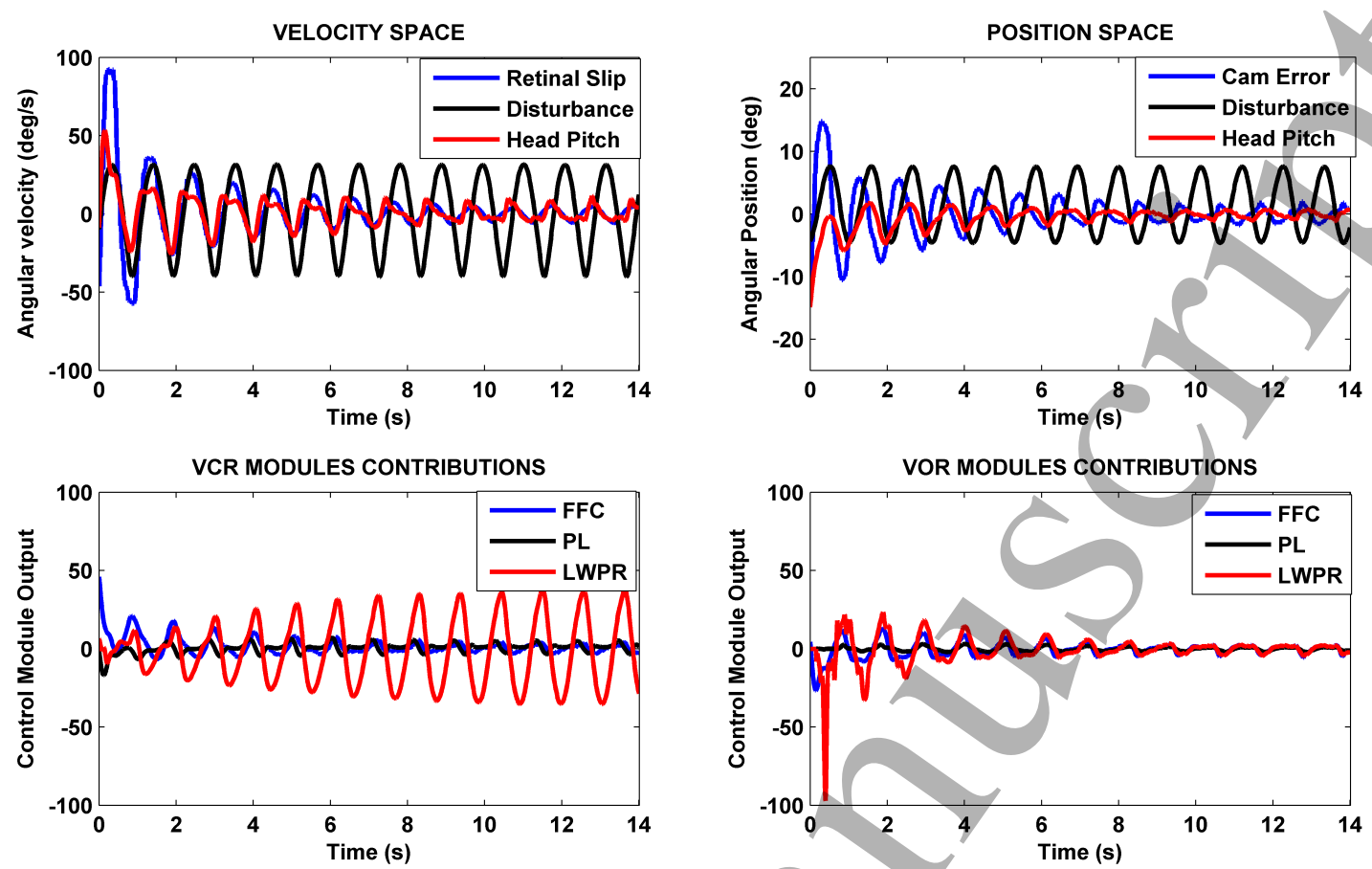

Figure 4: Execution trial for $f=1 \mathrm{~Hz}$. Results for the inertial readings and camera error in the velocity and position spaces are on the upper half, while the contributions of the feedforward controllers (FFC) and the internal model components (LWPR and PL), for both the VCR and VOR, are on the lower half.
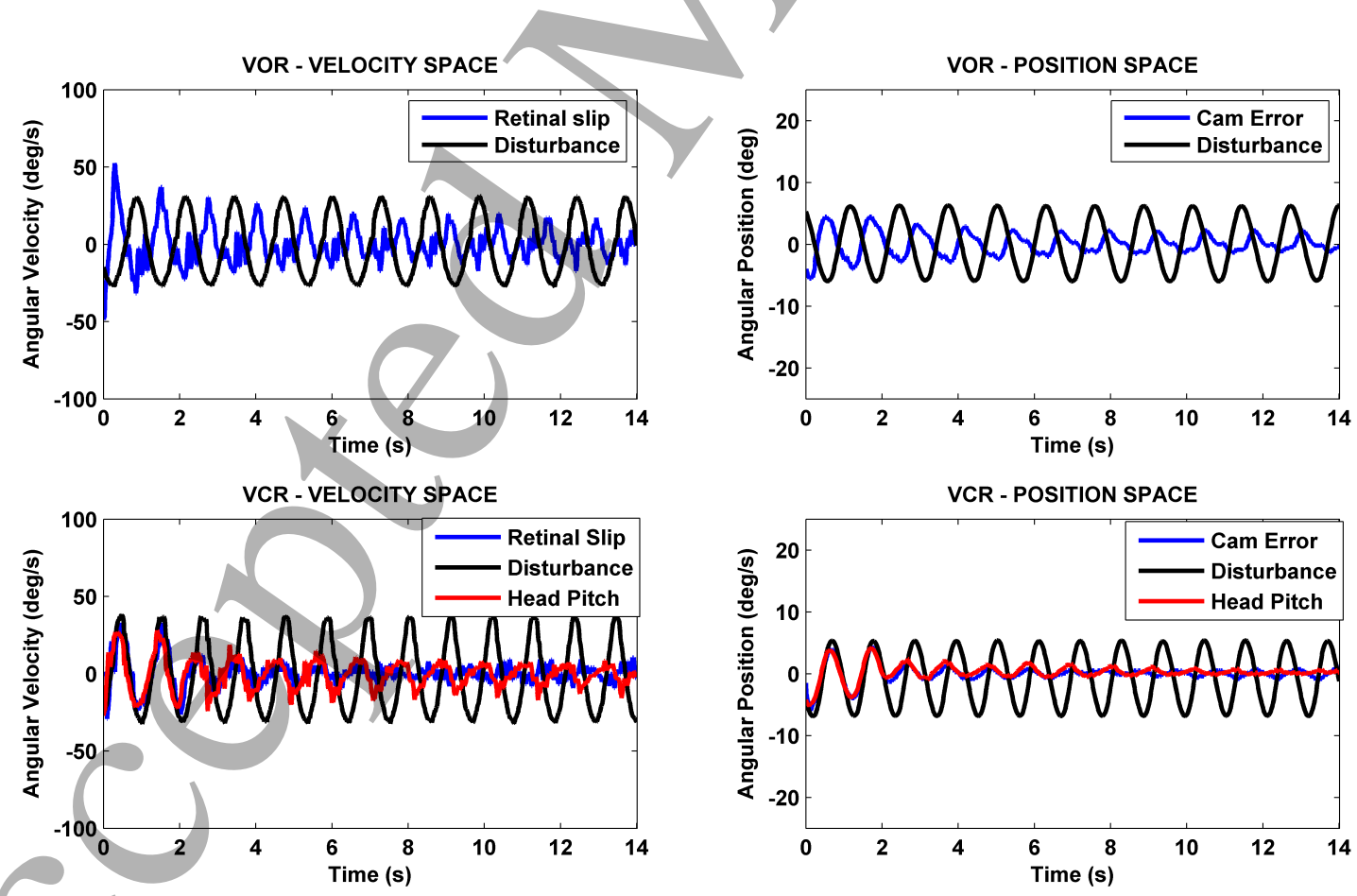

Figure 5: Execution trial for $f=1 \mathrm{~Hz}$ with only one of VCR or VOR subsystem active.

high frequencies. 

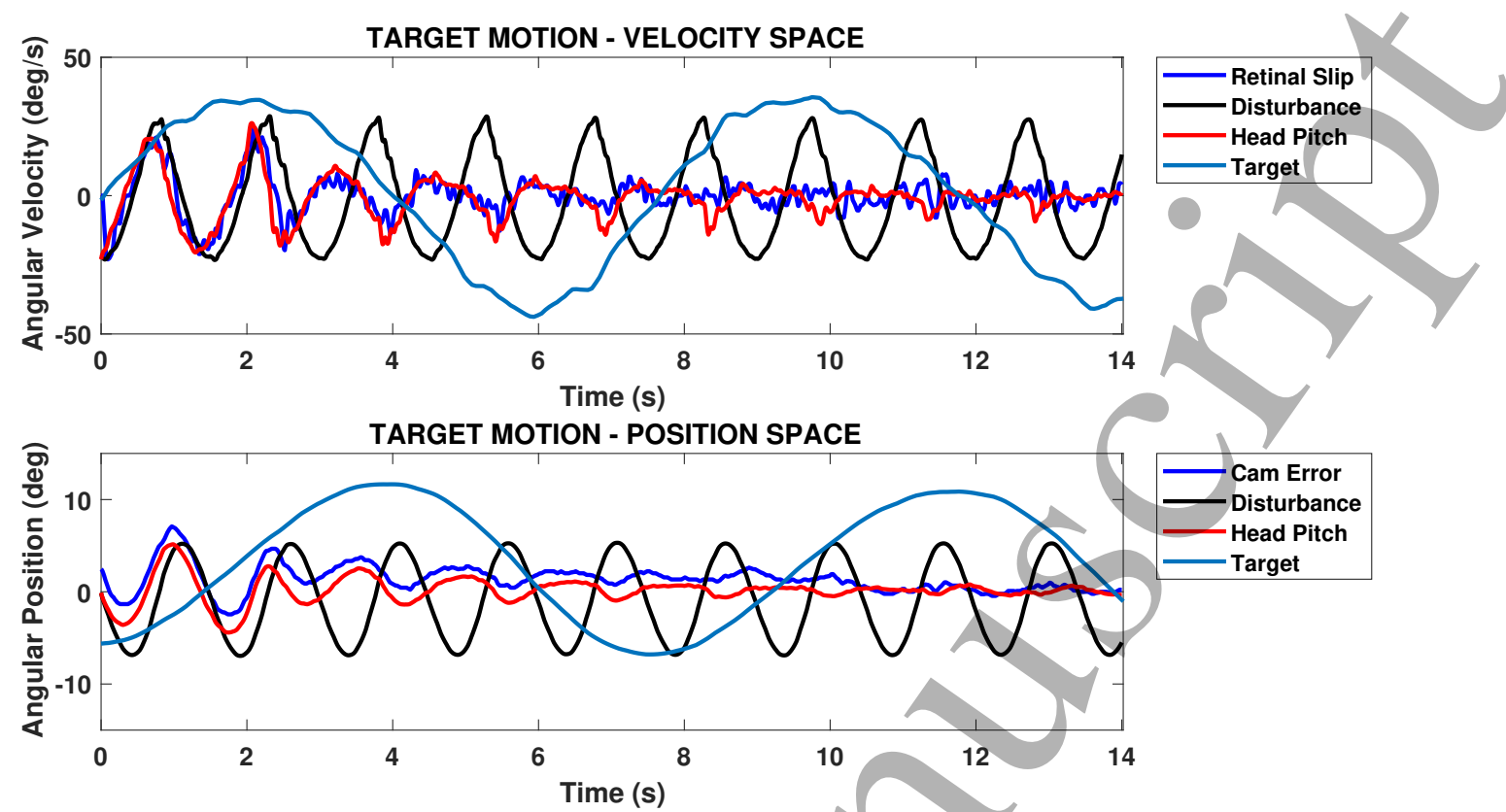

Figure 6: Execution trial for $f=1 \mathrm{~Hz}$ with elicitation of the OKR through a vertical movement of the target.
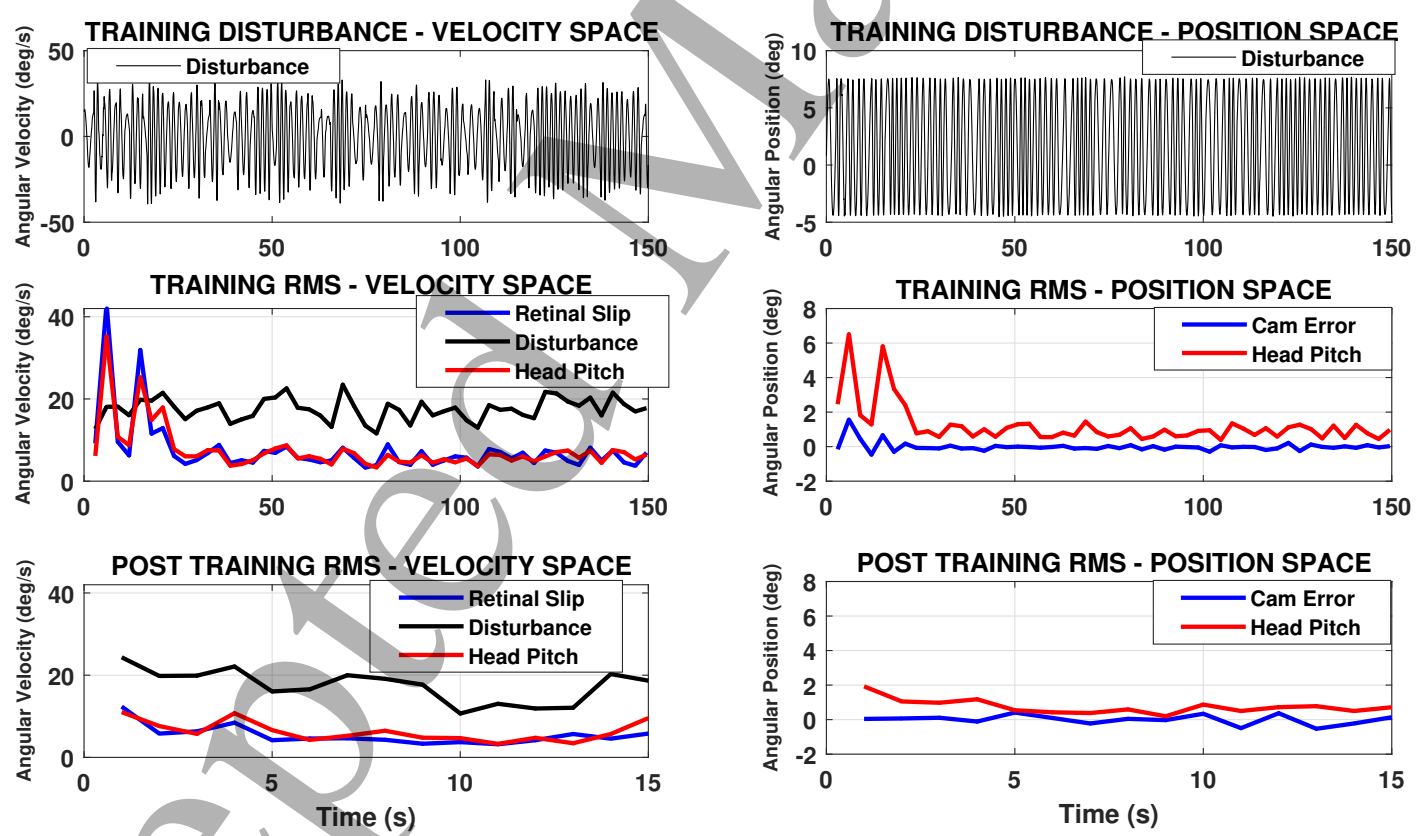

Figure 7: Offline learning with a randomly generated movement applied to the oscillating platform. The disturbance used for the training is shown on the top, the trend of the RMS of the disturbance and error signals during the training phase is show in the middle, while the behaviour of a similar trial, performed after training, is shown on the bottom.

An example of trial, for $f=1 \mathrm{~Hz}$, can be seen in Figure 9, where the disturbances, as well as the errors, on all rotational axes are shown. In accordance to the results observed for the oscillating platform, after an initial unstable phase, where learning occurs, both the inertial measurements and the camera errors decrease. While full stability of the camera image cannot be achieved due to the translation of the head caused 

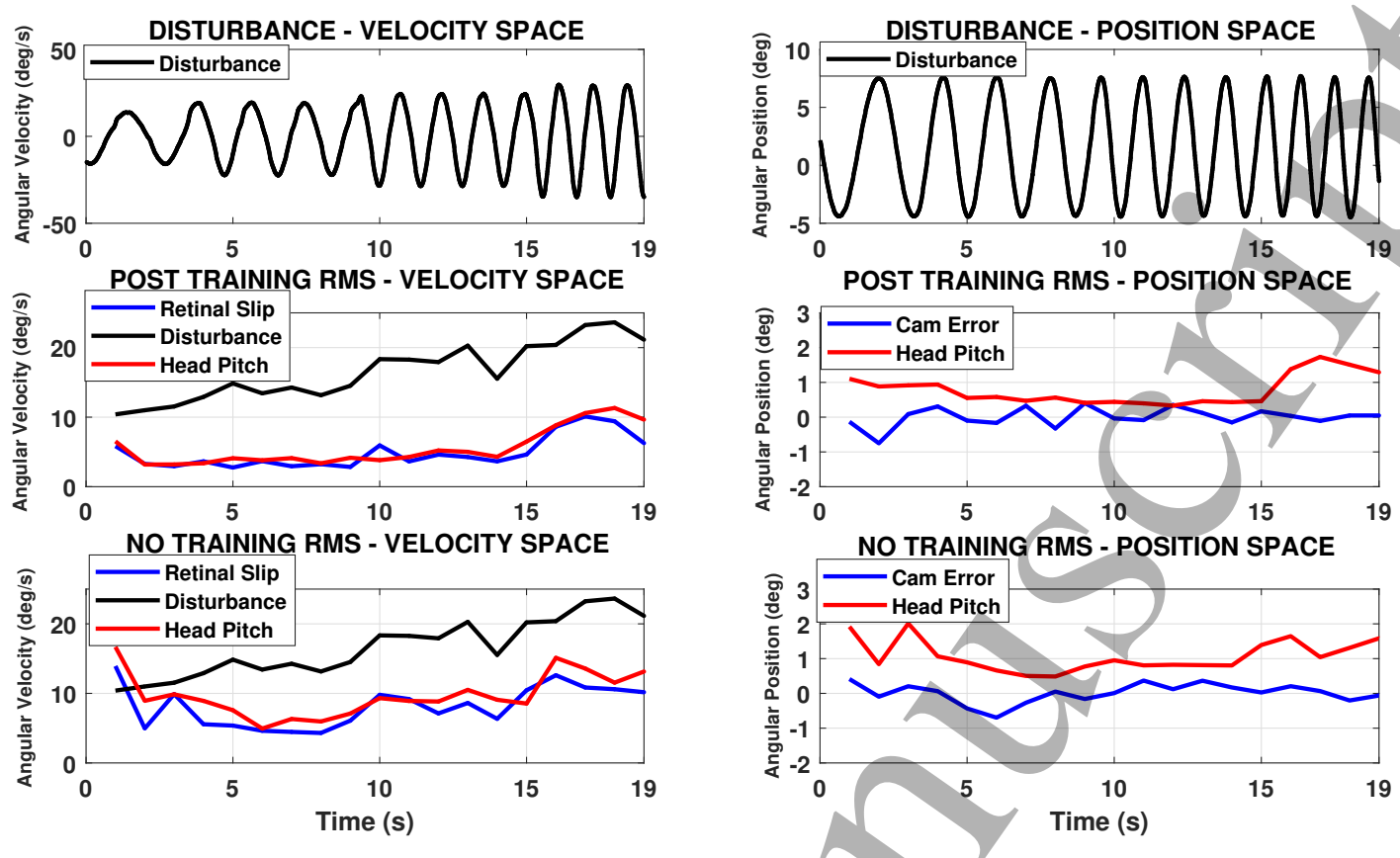

Figure 8: Comparison of the execution of the same trial by a controller trained with offline learning and another performing online learning. The frequency of the disturbance increases every 5 seconds.

Table 2: Results for disturbances provided by moving the robot torso on three axes, in simulation.

\begin{tabular}{|c||c|c|c|c|c|c|c|c|c|c|c|}
\hline$f$ & $\dot{d}_{p}$ & In $_{r}$ & In $_{r}$ & In $_{p}$ & In $_{p}$ & In $_{y}$ & In $_{y}$ & $u$ & $\dot{u}$ & $v$ & $\dot{v}$ \\
\hline 0.30 & 13.34 & 0.18 & 0.69 & 0.51 & 1.26 & 0.14 & 0.53 & 4.83 & 2.26 & 2.29 & 0.85 \\
0.50 & 22.32 & 0.31 & 1.80 & 0.39 & 1.94 & 0.13 & 0.53 & 4.83 & 3.81 & 2.29 & 1.59 \\
0.70 & 31.10 & 0.30 & 1.99 & 0.23 & 1.66 & 0.13 & 0.95 & 4.79 & 5.30 & 2.30 & 2.60 \\
0.85 & 37.69 & 0.30 & 2.25 & 0.51 & 3.13 & 0.17 & 1.35 & 4.80 & 7.56 & 2.27 & 3.22 \\
1.00 & 44.26 & 0.60 & 3.90 & 0.31 & 2.71 & 0.03 & 0.54 & 4.84 & 8.13 & 2.31 & 4.31 \\
1.20 & 52.83 & 0.13 & 1.96 & 0.42 & 3.89 & 0.09 & 1.92 & 4.84 & 11.21 & 2.29 & 4.59 \\
1.40 & 61.40 & 0.12 & 2.19 & 0.13 & 2.44 & 0.17 & 2.70 & 4.84 & 15.24 & 2.29 & 5.83 \\
1.50 & 65.52 & 0.53 & 5.95 & 0.56 & 6.22 & 0.30 & 4.32 & 4.83 & 17.54 & 2.30 & 6.18 \\
\hline
\end{tabular}

by the torso movements, the disturbance is greatly reduced by the controller.

\subsection{Stabilization during locomotion}

The effectiveness of the controller was also tested in a realistic situation by employing the biped humanoid robot SABIAN for a walking task consisting of 8 steps in a straight line (the step length was $160 \mathrm{~mm}$, step width $120 \mathrm{~mm}$, step time $1 \mathrm{~s}$ ). The walking control system of the WABIAN/SABIAN is composed by two modules phases (Lim, Yamamoto \& Takanishi 2002, Yamaguchi et al. 1999, Lim, Kaneshima \& Takanishi 2002). The first module uses a pattern generator that calculates the trajectory of the end-effectors (feet) and generates an ideal Zero Moment Point (ZMP) and the motion pattern of the lower limbs before the beginning of the walking task. The second module is active during the walking task, for the dynamic balance of the robot, providing corrections for leg and waist motions (Torso Position Control) according to the difference between the current and the ideal value of the ZMP positions (Lim et al. 2006, Yamaguchi et al. 1999, Hashimoto et al. 2012, Kang et al. 2012). In order to compute the camera error and the retinal slip, a target was placed in front of the robot, at the same height as the robot head. The target was kept static during these trials, thus the OKR module have been disabled. During a walking task, oscillations are produced on all three rotational axes, thus a stabilizing mechanism is needed for pitch, roll and yaw disturbances. However, due to the drifting of the IMU readings, it was not possible to perform stabilization on the yaw rotational axis. Therefore, the VCR was 

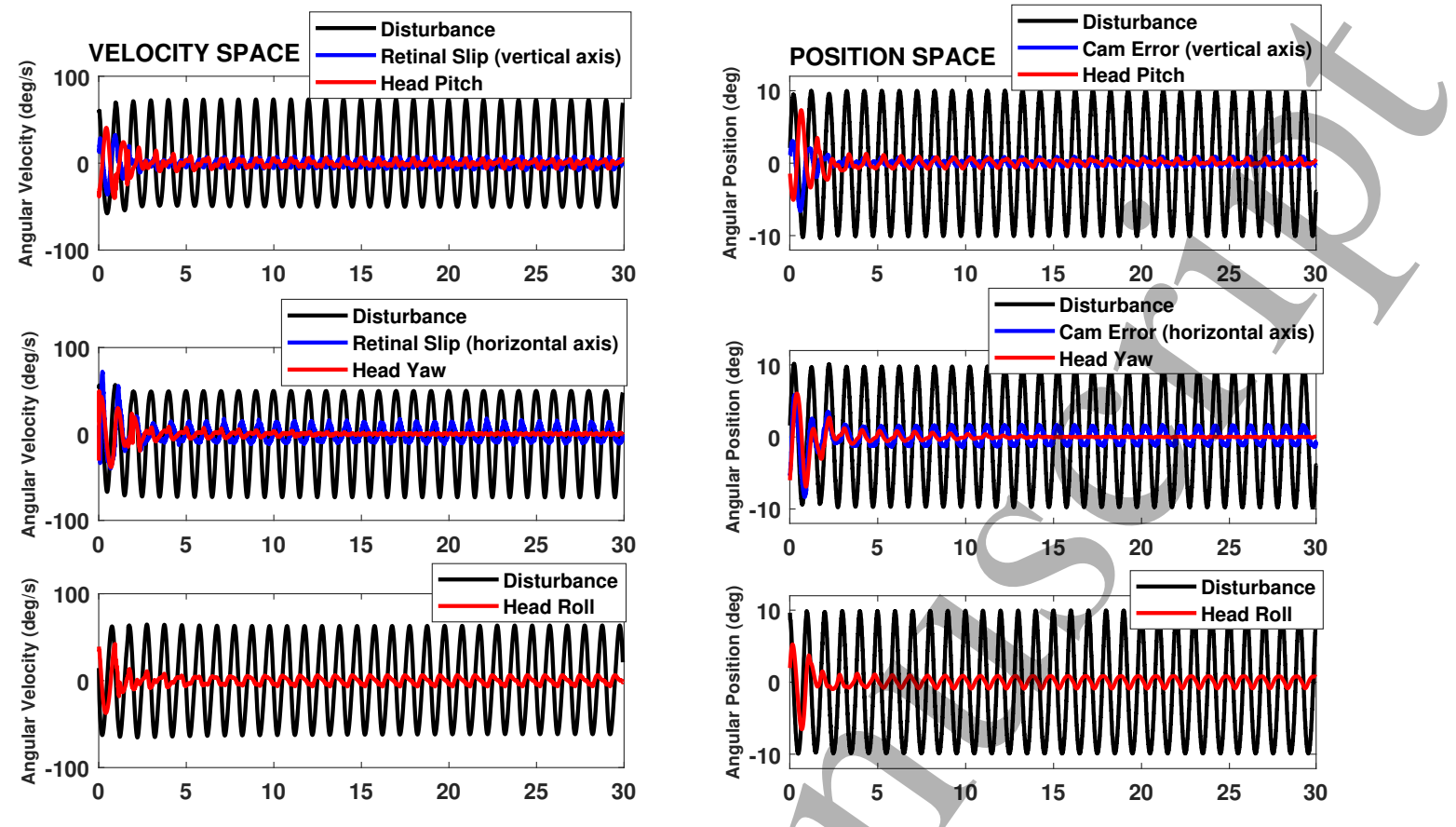

Figure 9: Stabilization of a sinusoidal motion with a frequency of $1 \mathrm{~Hz}$ applied on all three rotational axes, in simulation. Results for pitch, head and roll are on the top, middle and bottom rows, respectively.

only employed on the pitch and roll axes. Moreover the robot eyes cannot rotate around the roll axis, thus VOR compensation is provided only on the pitch axis.

Due to the shortness of the execution, each step lasts around one second for a total of 8 seconds, it was impossible for the internal models to have enough time to properly learn the motion dynamics. In addition, the disturbance in the velocity space had some considerable peaks probably due to the impulsive acceleration the head is subject to (see not stabilized gaze in Figure 10), that could slow down the learning. These two reasons make the online learning disadvantageous for such a short task. Thus, we employed the offline learning strategy by creating a training set consisting in inertial data recorded during 4 locomotion trials. All the collected data were glued together to create a new offline task lasting 32 seconds. Then, the controller was executed on such trial and the learnt internal models were saved. Finally, the modified version of the controller for the execution phase was employed on a new walking task. A comparison between a stabilized walk and a non stabilized one can be seen in Figure 10 . When the stabilization is employed the camera and inertial errors are signifieantly reduced in both the velocity and position spaces. Table 3 presents the results for two kinds of tests. In the first one the Torso Position Control (TPC) was active (as for the the trials used for the learning). In the other task the TPC was disabled producing a stronger disturbance on the head, but the gaze stabilization controller was able to effectively reject the disturbance also in this case. In particular, the RMS values for the camera errors, presented in Table $3\left(I n_{r}\right.$ and $I \dot{n}_{r}$ denote the inertial angular position and velocity along the roll axis), indicate a significant reduction of error while the controller is employed. In particular, the retinal slip value is lower than $4 \mathrm{deg} / \mathrm{s}$ thus demonstrating the effectiveness of the proposed stabilization mechanism on a realistic task.

Table 3: Comparison between camera and inertial RMS values in the stabilized and non-stabilized cases, in two different trials (with active and non-active TPC control).

\begin{tabular}{|c|c|c|c|c|c|c|c|c|}
\hline tr. & stab. & $\mathrm{TPC}$ & $I n_{p}$ & $\operatorname{In}_{p}$ & $I n_{r}$ & $I n_{r}$ & $v$ & $\dot{v}$ \\
\hline \multirow{2}{*}{1} & yes & yes & 0.19 & 1.62 & 0.33 & 1.46 & 0.29 & 3.23 \\
& no & yes & 0.47 & 3.06 & 0.61 & 2.59 & 0.76 & 6.58 \\
\hline \multirow{2}{*}{2} & yes & no & 0.38 & 1.68 & 0.88 & 2.84 & 0.27 & 3.34 \\
& no & no & 1.91 & 5.85 & 0.94 & 4.12 & 0.78 & 8.20 \\
\hline
\end{tabular}

\section{Conclusions}

In this work we present a complete controller for the stabilization of the gaze that is based on the coordination of VCR, VOR and OKR in conjunction with cerebellar internal models and we validate it through an implementation on a humanoid robotic platform. The model was tested on a robotic 
WALKING TASK - VELOCITY SPACE
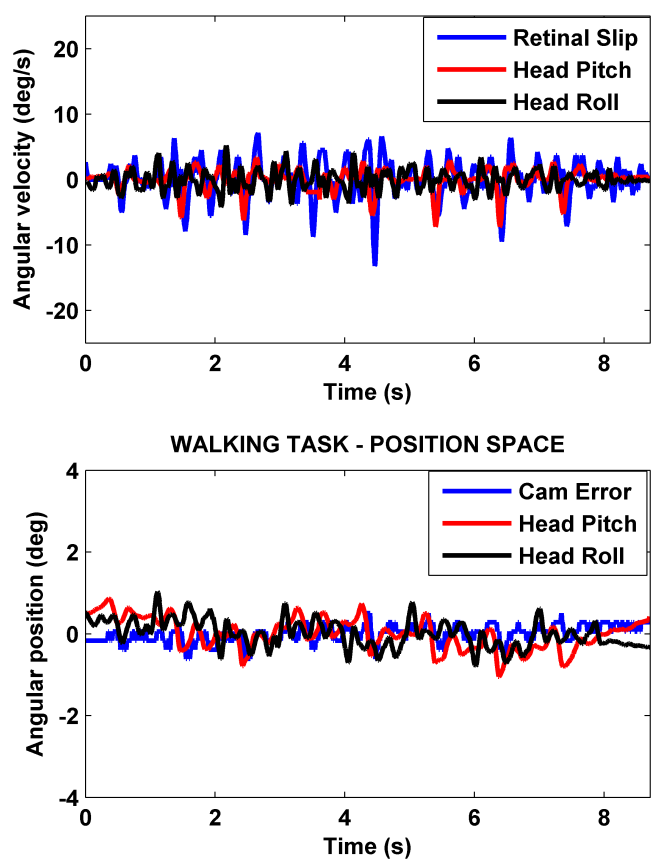

WALKING TASK - VELOCITY SPACE - NO STABILIZATION

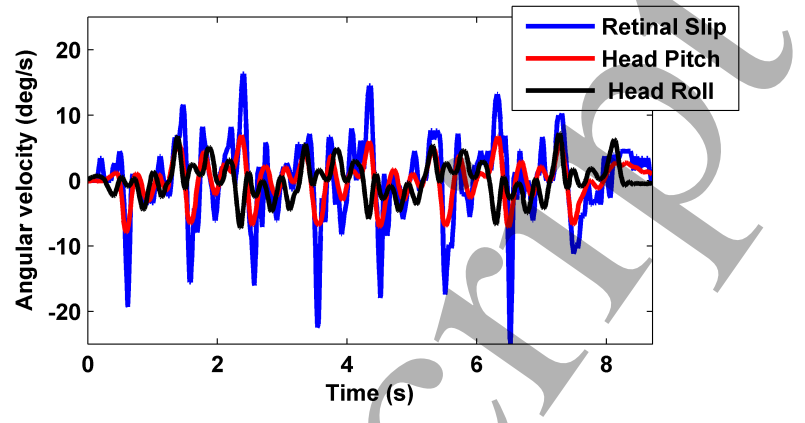

WALKING TASK - POSITION SPACE - NO STABILIZATION

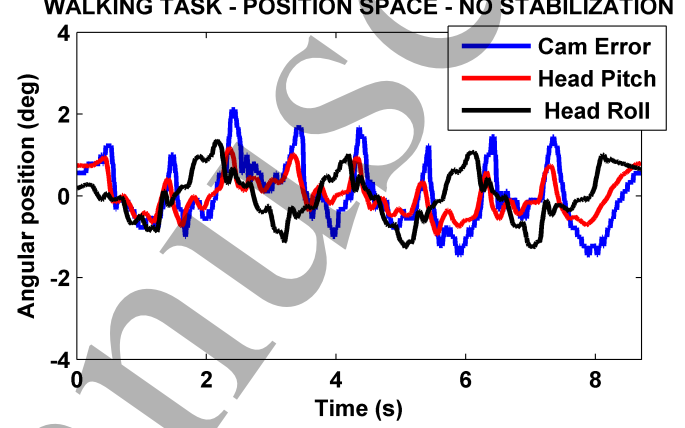

Figure 10: Stabilization during a straight walking task (with active TPC control), comparison between stabilized and non stabilized gaze.

head mounted on an oscillating platform capable of increase the learning times, therefore it should be only generating periodic disturbances and on a complete activated if its contribution could really be helpful in humanoid robot during a locomotion task. Results on the oscillating platform show that the model is able to keep the camera image stable while the disturbance is applied. The coordination of the VCR and VOR components proves beneficial for the overall performances and the controller is able to cope with drifts of the camera image thanks to the OKR subsystem. Moreover, tests performed during robotic locomotion trials prove the effectiveness of the approach in realistic scenarios. Experiments also shown that the internal models do not have to be learnt online during the execution of the task but can be generated by employing offline training and then stored to be used on real task. The model still has some limitations, and could be improved in some aspects. Due to the fact that only rotational information is used by the controller, disturbance produced by linear translations cannot be compensated. However, the controller could be in principle be extended to take also these inputs into account. Another simplification introduced was to align all sensory feedback in time introducing a fixed delay. In a more biologically accurate system, the delays could be different for every sensory pathway and could also be non constant. Enabling the OKR in some circumstances can significantly stabilizing the camera image. A possible improvement in this direction could be an automatic switching mechanism that enable the OKR when its contribution is required. Overall, this work has proven that the coordination of the three aforementioned reflex can increase the performances of gaze stabilization on physical humanoid robotic platforms and than can provide a fundamental component for visually-guided locomotion.

\section{Acknowledgments}

This project has received funding from the European Union's Horizon 2020 Research and Innovation Programme under Grant Agreement No. 720270 (HBP SGA1).

\section{References}

Albus, J. S. (1971). A theory of cerebellar function, Mathematical Biosciences 10(1-2): 25-61.

Barnes, G. (1993). Visual-vestibular interaction in the control of head and eye movement: the role of visual feedback and predictive mechanisms, Progress in Neurobiology 41(4): 435-472.

Beira, R., Lopes, M., Praga, M., Santos-Victor, J., Bernardino, A., Metta, G., Becchi, F. \& Saltarén, R. (2006). Design of the robot-cub (icub) head, Proc. IEEE International 
A comprehensive gaze stabilization controller based on cerebellar internal models

Conference on Robotics and Automation (ICRA'06), IEEE, pp. 94-100.

Bloomberg, J., Reschke, M., Huebner, W. \& Peters, B. (1992). The effects of target distance on eye and head movement during locomotion, Annals of the New York Academy of Sciences 656(1): 699-707.

Collewijn, H., Martins, A. \& Steinman, R. (1981). Natural retinal image motion: Origin and change, Annals of the New York Academy of Sciences 374(1): 312-329.

Cullen, K. E. (2012). The vestibular system: multimodal integration and encoding of self-motion for motor control, Trends in neurosciences 35(3): 185-196.

Cullen, K. E. \& Roy, J. E. (2004). Signal processing in the vestibular system during active versus passive head movements, Journal of Neurophysiology 91(5): 19191933.

Falotico, E., Cauli, N., Hashimoto, K., Kryczka, P., Takanishi, A., Dario, P., Berthoz, A. \& Laschi, C. (2012). Head stabilization based on a feedback error learning in a humanoid robot, Proceedings IEEE International Workshop on Robot and Human Interactive Communication, 2012 IEEE International Conference on, pp. 449-454.

Falotico, E., Cauli, N., Kryczka, P., Hashimoto, K., Berthoz, A., Takanishi, A., Dario, P. \& Laschi, C. (2017). Head stabilization in a humanoid robot: models and implementations, Autonomous Robots 41(2): 349-365.

Falotico, E., Laschi, C., Dario, P., Bernardin, D. \& Berthoz, A. (2011). Using trunk compensation to model head stabilization during locomotion, IEEE-RAS International Conference on Humanoid Robots, pp. 440445.

Forbes, P. A., de Bruijn, E., Schouten, A. C., van der Helm, F. C. \& Happee, R. (2013). Dependency of human neck reflex responses on the bandwidth of pseudorandom anterior-posterior torso perturbations, Experimental brain research 226(1): 1-14.

Forbes, P. A., Siegmund, G. P., Schouten, A. C. \& Blouin, J.-S. (2014). Task, muscle and frequency dependent vestibular control of posture, Frontiers in integrative neuroscience $\mathbf{8}$.

Franchi, E., Falotico, E., Zambrano, D., Muscolo, G., Marazzato, L., Dario, P. \& Laschi, C. (2010). A comparison between two bio-inspired adaptive models of vestibulo-ocular reflex (VOR) implemented on the iCub robot, 2010 10th IEEE-RAS International Conference on Humanoid Robots, Humanoids 2010, pp. 251-256.

Galiana, H. L. \& Outerbridge, J. S. (1984). A bilateral model for central neural pathways in vestibuloocular reflex, Journal of neurophysiology 51(2): 210-241.

Gay, S., Santos-Victor, J. \& Ijspeert, A. (2013). Learning robot gait stability using neural networks as sensory feedback function for central pattern generators, Intelligent Robots and Systems (IROS), 2013 IEEE/RSJ International Conference on, pp. 194-201.

Goldberg, J. M. \& Cullen, K. E. (2011). Vestibular control of the head: possible functions of the vestibulocollic reflex, Experimental brain research 210(3-4): 331-345.

Gomi, H. \& Kawato, M. (1992). Adaptive feedback control models of the vestibulocerebellum and spinocerebellum, Biological cybernetics 68(2): 105-114.

Green, A. M. \& Angelaki, D. E. (2004). An integrative neural network for detecting inertial motion and head orientation, Journal of neurophysiology 92(2): 905-925.

Habra, T. \& Ronsse, R. (2016). Gaze stabilization of a humanoid robot based on virtual linkage, Biomedical Robotics and Biomechatronics (BioRob), 2016 6th IEEE International Conference on, IEEE, pp. 163-169.

Hashimoto, K., Kang, H.-J., Nakamura, M., Falotico, E., Lim, H.-O., Takanishi, A., Laschi, C., Dario, P. \&
Berthoz, A. (2012). Realization of biped walking on soft ground with stabilization control based on gait analysis, Intelligent Robots and Systems (IROS), 2012 IEEE/RSJ International Conference on, pp. 2064-2069.

Itō, M. (1984). The cerebellum and neural control, Raven Pr.

Ito, M. (2000). Mechanisms of motor learning in the cerebellum1, Brain Research 886(12): 237 - 245.

Ito, M., Nisimaru, N. \& Yamamoto, M. (1977). Specific patterns of neuronal connexions involved in the control of the rabbit's vestibulo-ocular reflexes by the cerebellar flocculus., The Journal of Physiology 265(3): 833-854.

Kang, H.-J., Hashimoto, K., Nishikawa, K., Falotico, E., Lim, H.-O., Takanishi, A., Laschi,/C., Dario, P. \& Berthoz, A. (2012). Biped walking stabilization on soft ground based on gait analysis, Biomedical Robotics and Biomechatronics (BioRob), 2012 4th IEEE RAS EMBS International Conference on, pp. 669-674.

Kavanagh, J., Barrett, R. \& Morrison, S. (2006). The role of the neck and trunk in facilitating head stability during walking, Experimental brain research 172(4): 454-463.

Kryczka, P., Falotico, E., Hashimoto, K., Lim, H.-O., Takanishi, A., Laschi, C., Dario, P. \& Berthoz, A. (2012a). A robotic implementation of a bio-inspired head motion stabilization model on a humanoid platform, IEEE International Conference on Intelligent Robots and Systems, pp. 2076-2081.

Kryczka, P., Falotico, E., Hashimoto, K., Lim, H., Takanishi, A., Laschi, C., Dario, P. \& Berthoz, A. (2012b). Implementation of a human model for head stabilization on a humanoid platform, Proceedings of the IEEE RAS and EMBS International Conference on Biomedical Robotics and Biomechatronics, pp. 675-680.

C. G., Honrubia, V., Jenkins, H., Baloh, R. \& Yee, R. (1978). Linear model for visual-vestibular interaction., Aviation, space, and environmental medicine.

Lenz, A., Balakrishnan, T., Pipe, A. G. \& Melhuish, C. (2008). An adaptive gaze stabilization controller inspired by the vestibulo-ocular reflex, Bioinspiration 83 biomimetics 3(3): 035001.

Lim, H.-O., Kaneshima, Y. \& Takanishi, A. (2002). Online walking pattern generation for biped humanoid robot with trunk, Robotics and Automation, 2002. Proceedings. ICRA'02. IEEE International Conference on, Vol. 3, IEEE, pp. 3111-3116.

Lim, H.-O., Ogura, Y. \& Takanishi, A. (2006). Dynamic locomotion and mechanism of biped walking robot, 2006 SICE-ICASE International Joint Conference, pp. 34843489 .

Lim, H.-O., Yamamoto, Y. \& Takanishi, A. (2002). Stabilization control for biped follow walking, Advanced Robotics 16(4): 361-380.

Manzoni, D., Andre, P., d'Ascanio, P. \& Pompeiano, O. (1994). Depression of the vestibulospinal reflex adaptation by intravermal microinjection of gaba-a and gaba-b agonists in the cat, Archives italiennes de biologie 132(4): 243269.

Marcinkiewicz, M., Kaushik, R., Labutov, I., Parsons, S. \& Raphan, T. (2009). Learning to stabilize the head of a quadrupedal robot with an artificial vestibular system, Robotics and Automation, 2009. ICRA'09. IEEE International Conference on, IEEE, pp. 25122517.

Marr, D. \& Thach, W. T. (1991). From the Retina to the Neocortex: Selected Papers of David Marr, Birkhäuser Boston, Boston, MA, chapter A Theory of Cerebellar Cortex, pp. 11-50.

Miles, F. A. \& Lisberger, S. G. (1981). Plasticity in the vestibulo-ocular reflex: a new hypothesis, Annual review of neuroscience 4(1): 273-299.

Mulavara, A. P., Verstraete, M. C. \& Bloomberg, J. J. (2002). 
Modulation of head movement control in humans during treadmill walking, Gait \& posture 16(3): 271-282.

Ogura, Y., Aikawa, H., Shimomura, K., Kondo, H., Morishima, A., Lim, H.-o. \& Takanishi, A. (2006). Development of a new humanoid robot wabian-2, Proceedings 2006 IEEE International Conference on Robotics and Automation, 2006. ICRA 2006., IEEE, pp. 76-81.

Panerai, F., Metta, G. \& Sandini, G. (2000). Visuo-inertial stabilization in space-variant binocular systems, Robotics and Autonomous Systems 30(1): 195-214.

Panerai, F. \& Sandini, G. (1998). Oculo-motor stabilization reflexes: integration of inertial and visual information, Neural Networks 11(7): 1191-1204.

Peng, G., Hain, T. \& Peterson, B. (1996). A dynamical model for reflex activated head movements in the horizontal plane, Biological cybernetics 75(4): 309-319.

Peterson, B. W., Choi, H., Hain, T., Keshner, E. \& Peng, G. C. (2001). Dynamic and kinematic strategies for head movement control, Annals of the New York Academy of Sciences 942(1): 381-393.

Porrill, J. \& Dean, P. (2007). Recurrent cerebellar loops simplify adaptive control of redundant and nonlinear motor systems, Neural Computation 19(1): 170-193.

Porrill, J., Dean, P. \& Stone, J. V. (2004). Recurrent cerebellar architecture solves the motor-error problem, Proceedings of the Royal Society of London-B 271(1541): 789-796.

Pozzo, T., Berthoz, A. \& Lefort, L. (1990). Head stabilization during various locomotor tasks in humans, Experimental Brain Research 82(1): 97-106.

Robinson, D. (1976). Adaptive gain control of vestibuloocular reflex by the cerebellum, Journal of Neurophysiology 39(5): 954-969.

Roncone, A., Pattacini, U., Metta, G. \& Natale, L. (2014). Gaze stabilization for humanoid robots: A comprehensive framework, 2014 IEEE-RAS International Conference on Humanoid Robots, IEEE, pp. 259-264.

Schmid, R., Buizza, A. \& Zambarbieri, D. (1980). A nonlinear model for visual-vestibular interaction during body rotation in man, Biological cybernetics 36(3): 143151.

Schmid, R., Stefanelli, M. \& Mira, E. (1971). Mathematical modelling: a contribution to clinical vestibular analysis, Acta oto-laryngologica 72(1-6): 292-302.

Schweigart, G., Mergner, T., Evdokimidis, I., Morand, S. \& Becker, W. (1997). Gaze stabilization by optokinetic reflex (okr) and vestibulo-ocular reflex (vor) during active head rotation in man, Vision Research 37(12): 1643-1652.

Shibata, T. \& Schaal, S. (2001). Biomimetic gaze stabilization based on feedback-error-learning with nonparametric regression networks, Neural Networks 14(2): 201-216.

Tikhanoff, V., Cangelosi, A., Fitzpatrick, P., Metta, G., Natale, L. \& Nori, F. (2008). An open-source simulator for cognitive robotics research: The prototype of the icub humanoid robot simulator, Proceedings of the 8th Workshop on Performance Metrics for Intelligent Systems, PerMIS '08, ACM, New York, NY, USA, pp. $57-61$.

URL: http://doi.acm.org/10.1145/1774674.1774684

Tolu, S., Vanegas, M., Garrido, J. A., Luque, N. R. \& Ros, E. (2013). Adaptive and predictive control of a simulated robot arm, Int. J. Neural Syst. 23(3).

Tolu, S., Vanegas, M., Luque, N. R., Garrido, J. A. \& Ros, E. (2012). Bio-inspired adaptive feedback error learning architecture for motor control, Biological Cybernetics 106(8-9): 507-522.

Vannucci, L., Falotico, E., Tolu, S., Dario, P., Lund, H. H. \& Laschi, C. (2016). Eye-head stabilization mechanism for a humanoid robot tested on human inertial data, Lecture Notes in Computer Science (including subseries Lecture
Notes in Artificial Intelligence and Lecture Notes in Bioinformatics) 9793: 341-352.

Vannucci, L., Tolu, S., Falotico, E., Dario, P., Lund, H. H. \& Laschi, C. (2016). Adaptive gaze stabilization through cerebellar internal models in a humanoid robot, Proceedings of the IEEE RAS and EMBS International Conference on Biomedical Robotics and Biomechatronics, Vol. 2016-July, pp. 25-30.

Vijayakumar, S. \& Schaal, S. (2000). Locally weighted projection regression: Incremental real time learning in high dimensional space, ICML '00: Proceedings of the Seventeenth International Conference on Machine Learning, Morgan Kaufmann Publishers Inc., San Francisco, CA, USA, pp. 1079-1086.

Viollet, S. \& Franceschini, N. (2005). A high speed gaze control system based on the vestibulo-ocular reflex, Robotics and Autonomous Systems 50(4): 147-161.

Yamada, H., Mori, M. \& Hirose, S. (2007). Stabilization of the head of an undulating snake-like robot, Intelligent Robots and Systems (IROS), 2007 IEEE/RSJ International Conference on, pp. 3566-3571.

Yamaguchi, J., Soga, E., Inoue, S. \& Takanishi, A. (1999). Development of a bipedal humanoid robot-control method of whole body cooperative dynamic biped walking, Robotics and Automation, 1999. Proceedings. 1999 IEEE International Conference on, Vol. 1, IEEE, pp. 368-374.

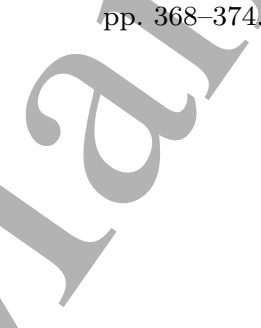

